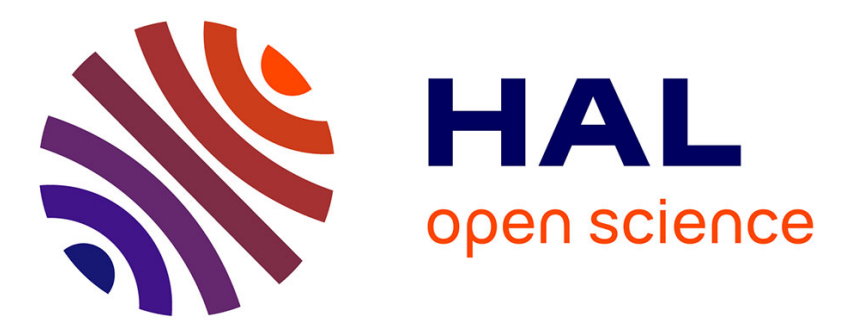

\title{
A New Observer for Range Identification in Perspective Vision Systems
}

Victor Gibert, Laurent Burlion, Abdelhamid Chriette, Josep Boada, Franck

Plestan

\section{> To cite this version:}

Victor Gibert, Laurent Burlion, Abdelhamid Chriette, Josep Boada, Franck Plestan. A New Observer for Range Identification in Perspective Vision Systems. Advances in Aerospace Guidance, Navigation and Control, pp.401-412, 2015, 10.1007/978-3-319-17518-8_23 . hal-01311370

\author{
HAL Id: hal-01311370 \\ https://hal.science/hal-01311370
}

Submitted on 18 May 2016

HAL is a multi-disciplinary open access archive for the deposit and dissemination of scientific research documents, whether they are published or not. The documents may come from teaching and research institutions in France or abroad, or from public or private research centers.
L'archive ouverte pluridisciplinaire HAL, est destinée au dépôt et à la diffusion de documents scientifiques de niveau recherche, publiés ou non, émanant des établissements d'enseignement et de recherche français ou étrangers, des laboratoires publics ou privés. 


\title{
A new observer for range identification in Perspective Vision Systems
}

\author{
V. GIBERT, L. BURLION, A. CHRIETTE, J. BOADA-BAUXELL, F. PLESTAN
}

\section{INTRODUCTION}

Automatic guidance of flying vehicle usually needs external information. GPS (Global Positionning System) is a worldwide technology which provides to the guided system its deviations with respect to its guidance objective. Nevertheless, this technology is not available everywhere (indoor evironment), everytime (in case of failure) and not precise enough to ensure critical operations as landing a civil aircraft. Current civil aircraft are able to land autonomously on a runway thanks to $I L S$ (Instrument Landing System) or differential GPS. However, these equipment are expensive and can fail. In frame of the future aircraft, manufacturers like AIRBUS company study the possibility to make aircraft landing everywhere (unequipped or unknown runway) without using informations from external systems.

In order to overcome the use of external needs, an embedded solution that provides deviations is needed. In parallel, image processing and camera technology have made a technological leap in the last decade. Hence, the use of a camera to perform visual servoing becomes an interesting solution to cope with precision and availability requirements.

Victor GIBERT

Airbus Operations S.A.S., Toulouse, France e-mail: victor.gibert@airbus.com

Laurent BURLION

ONERA, DCSD, Toulouse, France e-mail: laurent.burlion@onera.fr

Abdelhamid CHIRETTE

LUNAM Université, Ecole Centrale de Nantes - IRCCyN, Nantes, France e-mail: Abdelhamid.Chriette@irccyn.ec-nantes.fr

Josep BOADA-BAUXELL

Airbus Operations S.A.S., Toulouse, France e-mail: josep.boada-bauxell@airbus.com

Franck PLESTAN

LUNAM Université, Ecole Centrale de Nantes - IRCCyN, Nantes, France e-mail: Franck.Plestan@irccyn.ec-nantes.fr 
Visual servoing consists in using vision as a sensor in order to control the motion of the system. Tutorial in [3] explains the different ways to use visual servoing. Two main classes of visual servoing have been studied: the first and oldest one is Pose Based Visual Servoing $(P B V S)$ [1] whereas a more recent one is Image Based Visual Servoing (IBVS) [22]. In $P B V S$ scheme, vision is used to estimate the pose (position and attitude) of the camera. This estimation can be obtained by using more than one camera [23], by knowing dimensions of seen objects [14] or by taking into account the camera motions [4][6][7][12][8][15][16]. Once the estimate is complete, standard guidance laws can be set up by using estimated deviations.

On the other side, IBVS scheme expresses the objective and the current state in the image frame. This scheme can use, for example, the full image for an homography comparison [13][21] or is directly using the measurement of visual features coordinates [2][5][11][18] and make the current features match with their corresponding desired ones on the image plane.

IBVS takes advantage on PBVS because of the overcoming of estimation process and is less impacted by calibration errors. However, PBVS takes advantage on IBVS because it allows to use existing and certified guidance laws; in IBVS, new guidance laws need to be designed. In PBVS, stereo-vision appears difficult in this case of study because the distance from runway is important, calibration must be very precise and not impacted by vibrations and two camera bring weight and space problem. This paper considers a generic runway whose size and markers are not known: geometric reconstruction solutions using these informations can not then be applied. If the motion of the aircraft is known, the use of dynamics of visual features between several images is sufficient to estimate deviations w.r.t. the runway.

Among these visual servoing strategies, $P B V S$ using known motions appears to be a potential candidate for aircraft landing; thus, estimated deviations are used as measurement in order to guide the aircraft in final approach. Actually, aircraft dynamic can be considered always known thanks to IRS sensors availability.

The aim of the article is to estimate the deviations of the camera w.r.t. to the runway. In this purpose, the single available informations are the knowledge on rational and translational velocities provided by inertial sensors and visual measurement. The visual informations, provided by image processing algorithms, correspond to the perspective projection of a $3 \mathrm{D}$ point in the image plane.

The three main contributions of this paper can be presented as follows : a new nonlinear observer solution is proposed based on state coordinates transformation; a comparison with previously published solutions is made on a common example of literature [4]; and the proposed estimator has been applied on a realistic scenario corresponding to a civil aircraft landing. The paper shows the advantages of the new range identification estimator compared with previous solutions. First, the proposed solution provides a general observer formulation for non-linear problem with a generic correction term. This correction term admits several type of non-linear estimators. Second, using an expression in a transformed state coordinates renders the observer design simple compared with [16]. Compared with previous solutions 
[4][8][15], the dynamics of estimates is easy to control. Finally, the proposed solution presents good robustness properties with respect to noisy measurement.

The paper is organized as follows. Section 2 presents a standard range identification formulation. Then, Section 3 proposes a new pose estimation method with detailed observability analysis and design method of the observer. Next, section 4 presents results obtained on a standard example which are presented and compared with other existing solutions. Results obtained with the proposed observer on a realistic aircraft motion during landing phases are also presented. Finally, section 5 concludes this paper.

\section{Problem statement}

The problem under interest consists in using an embedded monocular camera in order to estimate three-dimensional deviations w.r.t. a point of interest from twodimensional image measurement.

Denoting $\boldsymbol{x}$ the three-dimensional coordinates of a point attached to the ground express in the camera frame $\mathscr{C}$, its dynamics reads as

$$
\dot{\boldsymbol{x}}=\underbrace{\left[\begin{array}{lll}
a_{11}(t) & a_{12}(t) & a_{13}(t) \\
a_{21}(t) & a_{22}(t) & a_{23}(t) \\
a_{31}(t) & a_{32}(t) & a_{33}(t)
\end{array}\right]}_{\mathbf{A}} \boldsymbol{x}+\underbrace{\left[\begin{array}{l}
b_{1}(t) \\
b_{2}(t) \\
b_{3}(t)
\end{array}\right]}_{\mathbf{B}}
$$

with $\mathbf{A}$ and $\mathbf{B}$ respectively the rotational and translational motion matrices which can be time-varying and supposed known.

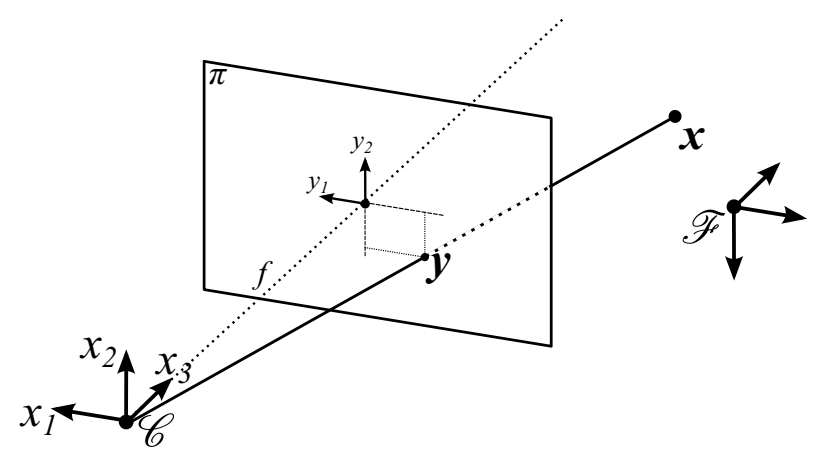

Fig. 1: Perspective projection of the runway in the camera plane. 
Assuming a calibrated pinhole camera model (see Figure 1), thanks to image processing algorithms, a perspective projection $\boldsymbol{y}=\left[\begin{array}{ll}y_{1} & y_{2}\end{array}\right]^{T}$ in the image plane $(\boldsymbol{\pi})$ is obtained from the unmeasurable state $x=\left[\begin{array}{lll}x_{1} & x_{2} & x_{3}\end{array}\right]^{T}$. The relationship between $y$ and $x$ reads as

$$
\boldsymbol{y}=C(\boldsymbol{x})=f\left[\frac{x_{1}}{x_{3}} \frac{x_{2}}{x_{3}}\right]^{T} .
$$

with $f$ the focal length of the camera ${ }^{1}$.

The objective of the following observer is to estimate the state coordinate $\left[\begin{array}{lll}x_{1} & x_{2} & x_{3}\end{array}\right]^{T}$ from the measurement of the image space coordinate $\left[\begin{array}{ll}y_{1} & y_{2}\end{array}\right]^{T}$ and the motions of the camera.

\section{A New Range Identification Technique}

Several approaches as [16][6][4] propose a range identification method in perspective system. This section proposes a new approach compared with the existing range identification methods in perspective system. The proposed solution allows an easy design of the correction term compared with previous observer and, as shown in a sequel, a better robustness versus noisy measurement. The new observer consists in using the canonical observability formulation with a state coordinate transformation that provides the capacity to easily design the observer. With this scheme, high gain or high order sliding-mode approaches could be applied.

\subsection{Observability Analysis}

Let us define the following function:

$$
\Psi(x)=\left[\begin{array}{l}
y_{1} \\
\dot{y}_{1} \\
y_{2}
\end{array}\right]=\left[\frac{x_{1}}{x_{3}}\left(\frac{\dot{x_{1}}}{x_{3}}\right) \frac{x_{2}}{x_{3}}\right]^{T}
$$

Consider $M_{x} \subset \mathbb{R}^{n}$ the operating physical domain in which $\boldsymbol{x}$ is evolving.

Definition 1. [17] The system (1)-(2) is locally observable (i.e., observable $\forall x \in M_{x}$ ) if $\Psi(x)$ is a state coordinates transformation, i.e., $\Psi(x)$ is invertible $\forall x \in M_{x}$.

Given the complexity of $\Psi$, it is difficult (even with formal computation software) to

\footnotetext{
${ }^{1}$ Without loss of generality, one can consider that the focal length $f=1$.
} 
analytically establish its invertibility. Thus, this latter will be numerically evaluated through the evaluation of its Jacobian $\frac{\partial \Psi}{\partial x}$.

Corollary 1 System (1)-(2) is locally observable in the sense of Definition 1 if

$$
\left(\frac{\partial \Psi}{\partial x}\right)_{x \in M_{x}} \text { invertible } \Leftrightarrow\left|\frac{\partial \Psi}{\partial x}\right|_{x \in M_{x}} \neq 0 .
$$

With the transformation $\Psi$ defined in (3), the observability condition is fulfilled if

$$
b_{1}-b_{3} y_{1}>0
$$

with $b_{i}$ from (1).

Remark 1 The choice for the function $\Psi$ is not unique. For example, it would be also possible to choose $\Psi=\left[\begin{array}{lll}y_{1} & y_{2} & \dot{y}_{2}\end{array}\right]^{T}$

\subsection{Observer design}

Given that $\Psi(x)$ is invertible under the proposed operating conditions (5), it defines a state coordinates transformation $\zeta=\Psi(x)$. Then, it is trivial to show that the nonlinear system (1)-(2) is locally equivalent to

$$
\dot{\zeta}=\underbrace{\left[\begin{array}{llll}
0 & 1 & 0 & 0 \\
0 & 0 & 0 & 0 \\
0 & 0 & 0 & 0
\end{array}\right]}_{F} \zeta+\underbrace{\left[\begin{array}{c}
0 \\
\Phi_{1}(\zeta) \\
\Phi_{2}(\zeta)
\end{array}\right]}_{\Phi(\zeta)}
$$

Proposition 1 An observer for system (6) reads as

$$
\dot{\hat{\zeta}}=F \hat{\zeta}+\Phi(\hat{\zeta})+\kappa(y, \hat{\zeta})
$$

with $\hat{\zeta}$ the estimated state of $\zeta$ and the function $\kappa(y, \hat{\zeta})$ called "correction term" and forcing $\hat{\zeta} \rightarrow \zeta$.

It is obvious that the correction term $\kappa(y, \hat{\zeta})$ is not unique and can be obtained by several different methods depending on the desired features (robustness, finite time convergence, etc.,). Note that the term $\kappa$ depends only on "known" variables, i.e. measurements $y$ and estimated state $\hat{\zeta}$. Given that estimation error dynamics reads as (with $e=\hat{\zeta}-\zeta$ )

$$
\dot{e}=F e+\Phi(\hat{\zeta})-\Phi(\zeta)+\kappa(y, \hat{\zeta})
$$

$\kappa(y, \hat{\zeta})$ has to force the observer to converge (exponentially or in a finite time) to the real system in spite of the initial error $e(0)$. From $\hat{\zeta}=\Psi(\hat{x})$, one gets 


$$
\dot{\hat{\zeta}}=\frac{\partial \Psi}{\partial \hat{x}} \dot{\hat{x}} \rightarrow \dot{\hat{x}}=\left[\frac{\partial \Psi}{\partial \hat{x}}\right]^{-1} \dot{\hat{\zeta}}
$$

Then, by a similar way than [19], an observer for system (1)-(2) reads as

$$
\dot{\hat{x}}=A \hat{x}+B+\left[\frac{\partial \Psi}{\partial x}\right]^{-1} \kappa(y, \hat{x})
$$

The structure of the observer having been defined, the correction term $\kappa(y, \hat{x})$ has to be designed. The proposed observer in (10) allows to choose among different correction term as high gain [10] or sliding mode observer [20] (more details in [9]). To provide a simple design of the correction term, the high gain observer has been chosen for this paper.

High-Gain Observer [10]: The observer (10) for the system (1)-(2) admits a correction term $\kappa(y, \hat{x})$ defined as

$$
\kappa(y, \hat{x})=\Lambda^{-1} K(y-C(\hat{x}))
$$

with

$$
\begin{gathered}
\Lambda=\left[\begin{array}{ccc}
\lambda_{1} & 0 & 0 \\
0 & \lambda_{1}^{2} & 0 \\
0 & 0 & \lambda_{2}
\end{array}\right] \\
K=\left[\begin{array}{cc}
K_{1} & 0 \\
K_{2} & 0 \\
0 & K_{3}
\end{array}\right] .
\end{gathered}
$$

and with $\lambda_{1}, \lambda_{2}, K_{1}, K_{2}$ and $K_{3}$ strictly positive constant so that $F-K C$ is Hurwitz.

The poles were specified in order to correspond to a characteristic polynomial with three parameters, $\alpha, \xi$ and $\omega$, as $(s+\alpha)\left(s^{2}+2 \xi \omega s+\omega^{2}\right)=0$. It remains to $K_{1}=2 \xi \omega, K_{2}=\omega^{2}$ and $K_{3}=\alpha$.

\section{Simulation Results}

\subsection{Academic example}

Numerical simulations are presented using the proposed observer (10). A comparison with existing approaches for range identification [4][6][8][16] allows assessing the performance of this new estimator solution. Consider the example given in example [4] for the affine system (1)-(2), with matrices $\mathbf{A}$ and $\mathbf{B}$ defined as

$$
\mathbf{A}=\left[\begin{array}{ccc}
-0.2 & 0.4 & -0.6 \\
0.1 & -0.2 & 0.3 \\
0.3 & -0.4 & 0.4
\end{array}\right] \text { and } \mathbf{B}=\left[\begin{array}{c}
0.5 \\
0.25 \\
0.3
\end{array}\right]
$$


with the initial conditions

$$
\left[\begin{array}{lll}
x_{1}(0) & x_{2}(0) & x_{3}(0)
\end{array}\right]^{T}=\left[\begin{array}{lll}
1 & 1.5 & 2.5
\end{array}\right]^{T}
$$

and

$$
\left[\begin{array}{lll}
\hat{x}_{1}(0) & \hat{x}_{2}(0) & \hat{x}_{3}(0)
\end{array}\right]^{T}=\left[\begin{array}{lll}
0.4 & 0.6 & 1
\end{array}\right]^{T}
$$

The desired rate of convergence of the estimate error is obtained with $\xi=0.99$ and $\omega=7$ corresponding to a 5\% response time of 1 seconds with strong damping and $\alpha=1$. These parameters roughly correspond to $\lambda=30$ in [16], to the same parameters than in [4] except $\delta_{i}=0.003$ and to the same parameters in [8] and in [6].

Simulation results for estimation of $\hat{x}_{3}$ with observers [4][6][8][16] in case of no measurement noise are shown in Figure 2.

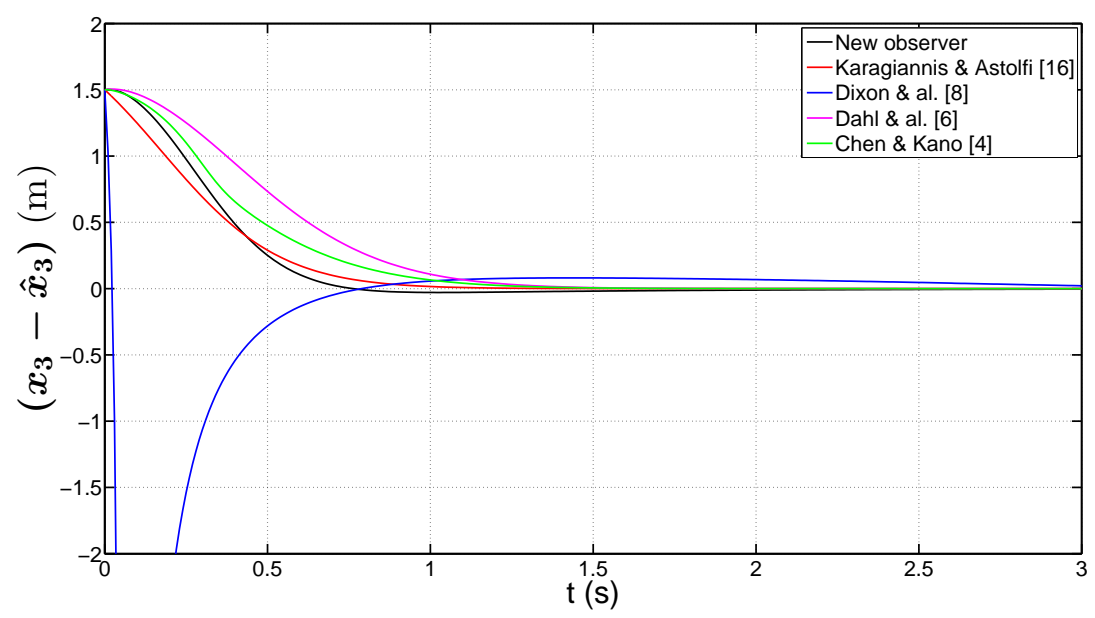

Fig. 2: Comparison between range identification methods $[4][6][8][16]$ and the proposed solution without noisy measurement.

Most observers have similar behavior except the range identification proposed by [8]. Note that, estimation error dynamics present a non-null tangent at the origint only with [16]. Taking into account the influence of observer design parameters on the error estimation dynamics, the proposed solution appears to be a good candidate to range identification problem in perspective systems.

To investigate the effects of noisy measurements on the estimation results, a $1 \%$ random noise of the signals is added on the measured informations $y$. Figure 3 shows the results for estimation of $\hat{y}_{3}$ under noisy conditions.

In presence of noise, the effects on estimations are not the same for all observers. Although the dynamic estimation is approximately the same for each estimator, the proposed solution is showing a very good robustness to noisy measurement com- 


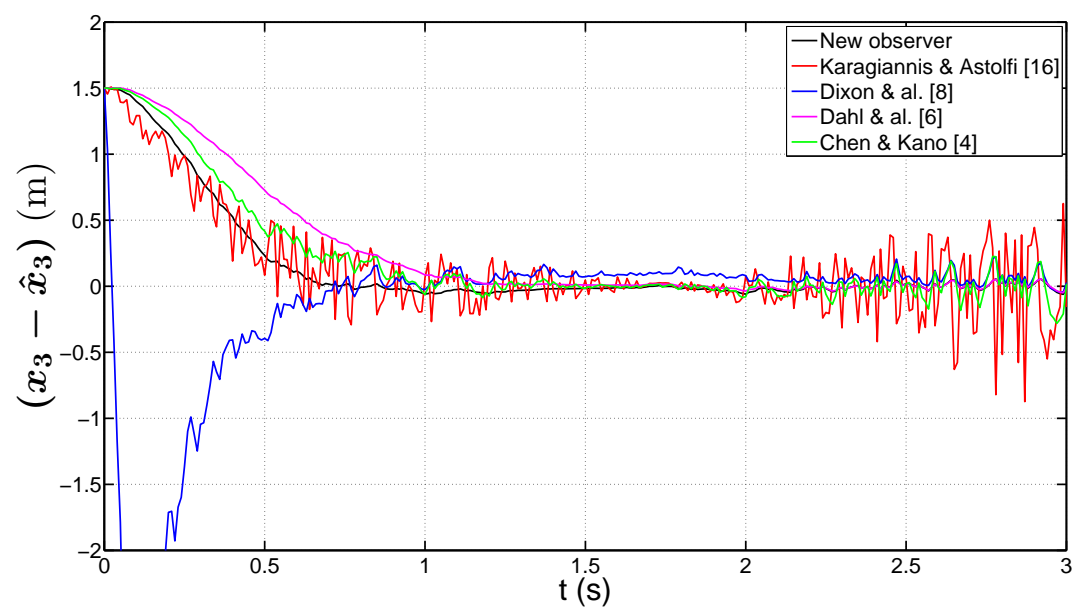

Fig. 3: Comparison between range identification methods [4][6][8][16] and the proposed solution in presence of noisy measurement.

pared with other observer. A similar robustness to noisy measurement is obtained with [6]. Indeed, this observer is also based on a coordinate transformation.

To conclude, the proposed observer is providing a new scheme for range identification in perspective vision system. Dynamics of the estimation rate could be easily tuned and robustness to noisy measurement appears to be one of the features of this estimation solution.

\subsection{Example based on a fixed wing civil aircraft}

Previous section results have been obtained on a very simple example with constant motion parameters. Hereafter, the proposed observer is applied on a realistic timevarying motion of a fixed wing civil aircraft. The coordinates of a tracking point $\boldsymbol{x}$ in the camera frame are expressed by

$$
\dot{\boldsymbol{x}}=\underbrace{\left[\begin{array}{ccc}
0 & -\omega_{z} & \omega_{y} \\
\omega_{z} & 0 & -\omega_{x} \\
-\omega_{y} & \omega_{x} & 0
\end{array}\right]}_{\mathbf{A}} \boldsymbol{x}+\underbrace{\left[\begin{array}{c}
v_{x}(t) \\
v_{y}(t) \\
v_{z}(t)
\end{array}\right]}_{\mathbf{B}}
$$

with $\omega_{i}$ and $V_{i}$ respectively the rotational and translational velocities expressed in the camera frame ${ }^{2}$.

${ }^{2}$ An embedded camera attached to the aircraft is used; here, the aircraft frame can be supposed merging with it. 
In final approach, the desired trajectory, named glide path, is ending on the runway at a 3D point $x$ (see Figure 4). The missing informations which need to be estimated are the deviations $x=\left[\begin{array}{lll}x_{1} & x_{2} & x_{3}\end{array}\right]^{T}$ w.r.t. the aircraft.

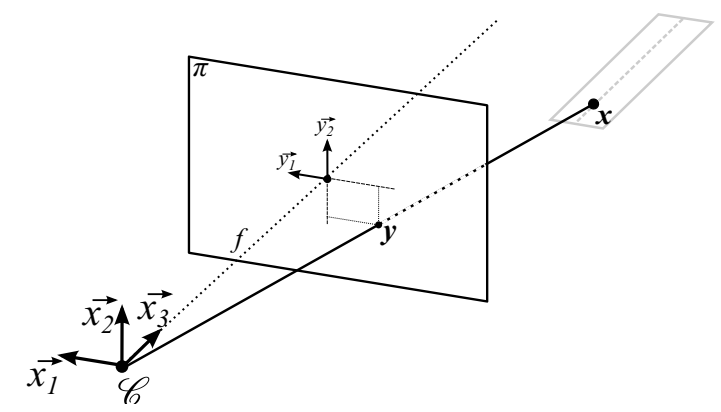

Fig. 4: Perspective projection of the glide path ending point on the runway $x$

Consider an aircraft, reaching the airport with a rough known position. At $5 \mathrm{~km}$ from the runway, one can consider the image processing able to deliver the perspective projection coordinates of $x$ in the image plane. The aircraft is neither aligned with the runway and nor already on the glide path. In the simulations, the aircraft will reach the desired trajectory and will track it during the end of the approach.

The observer will be initialized with wrong deviations $\left[\hat{x}_{1}(0) \hat{x}_{2}(0) \hat{x}_{3}(0)\right]$. Two initialised states will be simulated corresponding to a $\pm 50 \%$ error between the estimated and current state vectors. Figure 5 shows the trajectory followed by $x$ in the camera frame and the estimation of the 3D coordinates of $x$ during the landing with the two initialized states.

Simulation results confirm that the proposed observer provides a good estimation with a time varying motion of the camera. Estimation convergence is fast enough compared with the time to landing and the estimation error converges to zero.

Remark 2 Previous results have been obtained along a trajectory independent on the estimation results. In visual servoing, the estimated deviations feed the guidance law. Nevertheless, the estimated deviations of $x$, expressed in the camera frame, should be expressed in the inertial frame (attached to the runway). In this purpose, a change coordinate must be done using the rotational matrix $R=R_{\phi} R_{\theta} R_{\psi}$ which uses the orientation of the aircraft frame (i.e. camera frame) with the inertial frame. Note that the rotational matricies depends on the roll angle $\phi$, the pitch angle $\theta$ and the heading difference between the aircraft and the runway $\Delta_{\psi}$. The Inertial Measurement Unit (IMU) is providing $\phi$ and $\theta$ whereas $\Delta_{\psi}$ need to be measured. Particular visual features could be used to provide $\Delta_{\psi}$ as shown in appendix. 


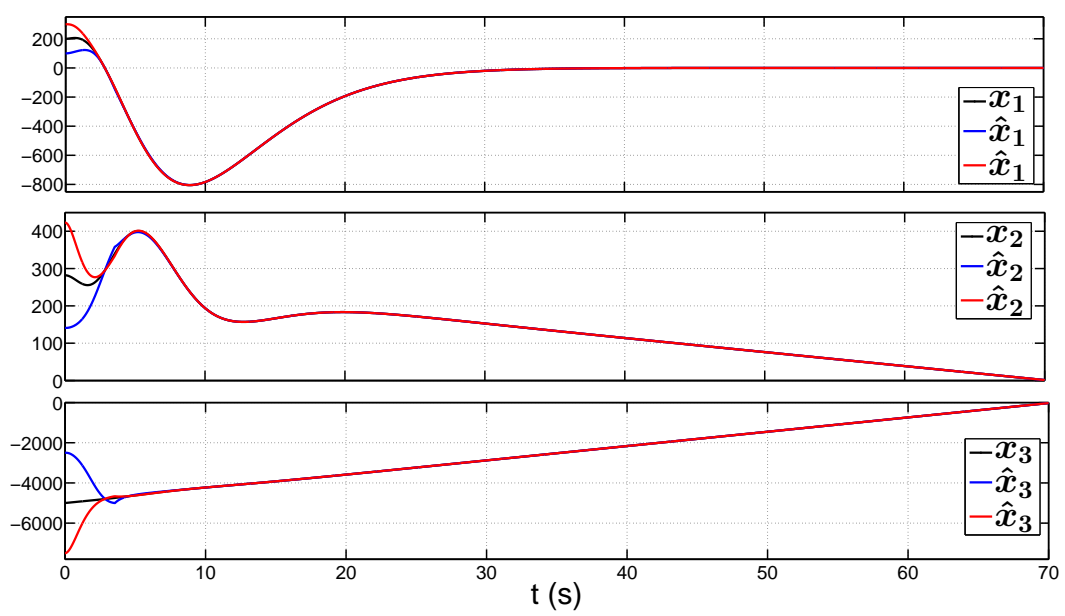

Fig. 5: Estimation results during a civil aircraft landing. Aircraft trajecory (in black), estimation with $-50 \%$ initial error (in blue) and estimation with $+50 \%$ initial error (in red).

\section{CONCLUSION AND FUTURE PERSPECTIVES}

This paper proposes a new pose estimation solution using a nonlinear observer. The presented solution provides good performances and appears to be more robust versus noisy measurement compared with previous solution. The proposed observer has been also applied on a realistic landing scenario.

Future works will focus on observability limitations during landing approach and calibrations errors effects. Furthermore, delays caused by image processing computation or numerical discretization caused by embedded computers will be taken into account.

\section{APPENDIX}

The measurement of $\Delta_{\psi}$ could be obtained from the image by using the visual feature $d_{F}$ (see Figure 6). Indeed, this visual feature depends only on $f$ (the focal length), $\phi, \theta$ and $\Delta_{\psi}$. It gives

$$
d_{F}=f\left(\frac{\tan \Delta_{\psi}}{\cos \theta}+\tan \phi \tan \theta\right)
$$

From (18), one can compute $\Delta_{\psi}$ with

$$
\Delta_{\psi}=\tan ^{-1}\left(\cos \theta\left(\frac{d_{F}}{f}-\tan \phi \tan \theta\right)\right) .
$$




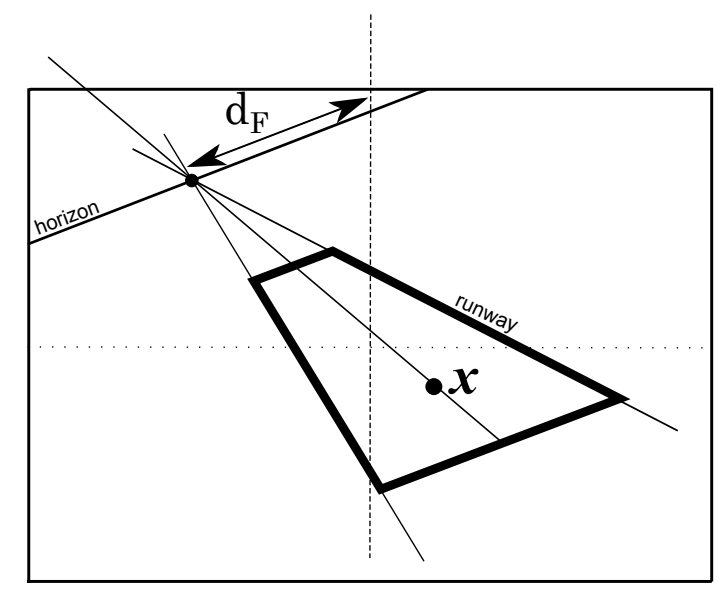

Fig. 6: Visual features $d_{F}$ corresponding to the distance between the vanishing point of runway side lines and the middle of the image along the horizon line.

\section{References}

1. Agin, G.: Computer vision systems for industrial inspection and assembly. Computer 13(5), $11-20(1980)$

2. Bourquardez, O., Chaumette, F.: Visual servoing of an airplane for alignment with respect to a runway. In: IEEE International Conference on Robotics and Automation. Roma, Italia (2007)

3. Chaumette, F., Hutchinson, S.: Visual servo control. i. basic approaches. Robotics Automation Magazine, IEEE 13(4), 82-90 (2006)

4. Chen, X., Kano, H.: A new state observer for perspective systems. IEEE Transactions on Automatic Control 47(4), 658-663 (2002)

5. Coutard, L., Chaumette, F., Pflimlin, J.M.: Automatic landing on aircraft carrier by visual servoing. In: Intelligent Robots and Systems (IROS), 2011 IEEE/RSJ International Conference on, pp. 2843-2848 (2011)

6. Dahl, O., Nyberg, F., Holst, J., Heyden, A.: Linear design of a nonlinear observer for perspective systems. In: IEEE International Conference on Robotics and Automation, pp. 429-435. Bareclona, Spain (2005)

7. De Luca, A., Oriolo, G., Giordano, P.: On-line estimation of feature depth for image-based visual servoing schemes. In: Robotics and Automation, 2007 IEEE International Conference on, pp. 2823-2828 (2007)

8. Dixon, W.E., Fang, D., Dawson, D.M., Flynn, T.J.: Range identification for perspective vision systems. IEEE Transactions on Automatic Control 48(12), 2232-2238 (2003)

9. El Tannoury, C., Moussaoui, S., Plestan, F., Romani, N., Pita-Gil, G.: Synthesis and application of nonlinear observers for the estimation of tire effective radius and rolling resistance of an automotive vehicle. Control Systems Technology, IEEE Transactions on 21(6), 2408-2416 (2013)

10. Gauthier, J.P., Hammouri, H., Othman, S.: A simple observer for nonlinear systems, application to bioreactors. IEEE Transactions on Automatic Control 37(6), 875-880 (1992)

11. Gibert, V., Puyou, G.: Landing of a transport aircraft using image based visual servoing. In: 9th IFAC Symposium on Nonlinear Control Systems, pp. 74-79. Toulouse, France (2013)

12. Giordano, P., De Luca, A., Oriolo, G.: 3d structure identification from image moments. In: IEEE International Conference on Robotics and Automation, pp. 93-100. Pasadena, California, USA (2008) 
13. Gonçalves, T., Azinheira, J., Rives, P.: Homography-based visual servoing of an aircraft for automatic approach and landing. In: IEEE International Conference on Robotics and Automation. Anchorage, Alaska, USA (2010)

14. Gui, Y., Guo, P., Zhang, H., Lei, Z., Zhou, X., Du, J., Yu, Q.: Airborne vision-based navigation method for uav accuracy landing using infrared lamps. Journal of Intelligent \& Robotic Systems 72(2), 197-218 (2013)

15. Jankovic, M., Ghosh, B.K.: Visually guided ranging from observations of points, lines and curves via an identifier based nonlinear observer. Systems and Control Letters 25(1), 63 - 73 (1995)

16. Karagiannis, D., Astolfi, A.: A new solution to the problem of range identification in perspective vision systems. IEEE Transactions on Automatic Control 50(12), 2074-2077 (2005)

17. Krener, A.J., Respondek, W.: Nonlinear observers with linearizable error dynamics. SIAM Journal on Control and Optimization 23(2), 197-216 (1985)

18. Le Bras, F., Hamel, T., Barat, C., Mahony, R.: Nonlinear image-based visual servo controller for automatic landing guidance of a fixed-wing aircraft. In: European Control Conference. Budapest, Hungary (2009)

19. Lebastard, V., Aoustin, Y., Plestan, F.: Estimation of absolute orientation for a bipedal robot: Experimental results. Robotics, IEEE Transactions on 27(1), 170-174 (2011)

20. Levant, A.: Higher-order sliding modes, differentiation and output-feedback control. International Journal of Control 76(9-10), 924-941 (2003)

21. de Plinval, H., Morin, P., Mouyon, P., Hamel, T.: Visual servoing for underactuated vtol uavs: a linear homography-based framework. International Journal of Robust and Nonlinear Control (2013)

22. Sanderson, A., Weiss, L.: Adaptive visual servo control of robots. In: A. Pugh (ed.) Robot Vision, International Trends in Manufacturing Technology, pp. 107-116 (1983)

23. Trisiripisal, P., Parks, M.R., Abbott, A.L., Liu, T., Fleming, G.A.: Stereo analysis for visionbased guidance and control of aircraft landing. In: 44th AIAA Aerospace Science Meeting and Exhibit. Reno, Nevada, USA (2006) 